\title{
Ganho de Peso da Desmama aos 12 Meses e Peso aos 12 Meses de Bovinos Nelore e Cruzas com Nelore
}

\section{Daniel Perotto ${ }^{2}$, Antonio Carlos Cubas $^{3}$, José Jorge dos Santos Abrahão ${ }^{4}$, Sílvio Carlos Mella ${ }^{5}$}

RESUMO - Foram analisados o ganho médio diário de peso da desmama aos 12 meses (GMD_D12) e o peso aos 12 meses de idade (P12M) de bovinos Nelore (N), Guzerá x N (GN), Red Angus x N (RN) e Marchigiana x N (MN), oriundos de um experimento de cruzamentos realizado na Estação Experimental do IAPAR de Paranavaí, produzidos por meio de inseminação artificial, nascidos no período de 1985 a 1997 em duas estações anuais de nascimento (janeiro a abril e julho a dezembro). Foi utilizado o método dos quadrados mínimos para análise de 634 observações de GMD_D12 e de P12M. Mês de nascimento do bezerro foi efeito significativo para GMD_D12 e para P12M. Para ambas as características, houve efeito relevante dos fatores ano de nascimento e sexo do bezerro, raça ou grupo genético, touro dentro de grupo genético e da interação grupo genético x sexo do animal. As médias dos quadrados mínimos e respecticos erros padrão, sempre na sequência N, GN, RN e MN foram : 0,247 $\pm 0,009 \mathrm{~kg} ; 0,287 \pm 0,010 \mathrm{~kg} ; 0,366 \pm 0,010 \mathrm{~kg}$ e 0,352 $\pm 0,012 \mathrm{~kg}$, para GMD_D12 e $176,82 \pm 1,86 \mathrm{~kg} ; 190,59 \pm 2,07 \mathrm{~kg} ; 223,30 \pm 2,31 \mathrm{~kg}$ e $214,12 \pm 2,31 \mathrm{~kg}$, para P12M.

Palavras-chave: gado de corte, cruzamentos, ganho de peso, peso pós-desmama

\section{Daily Gain from Weaning to Yearling Weight of Purebred and Crossbred Nellore Cattle}

\begin{abstract}
Average daily gain from weaning (GMD_12) to yearling weight (P12M) of Nellore, Guzerá x Nellore (GN), Red Angus x Nellore (RN), and Marchigiana x Nellore (MN) cattle, from a crossbreeding experiment conducted at the IAPAR Paranavaí Experimental Station, produced by artificial insemination, born between 1985 and 1997 within two annual birth seasons (January - April and July - December), were analyzed. The least squares method was utilized to study 634 observations of GMD_D12 and of P12M. Calf's birth month was a significant effect for GMD_D12 and for P12M. For both traits, there were relevant effects of the factors calf birth year and sex, genetic group or breed, sire within genetic group, and the interaction between the animal sex by genetic group. The least squares means and respective standard errors, always in the sequency N, GN, RN and MN were: $0.247 \pm 0.009 \mathrm{~kg}, 0.287 \pm 0.010 \mathrm{~kg}, 0.366 \pm 0.010 \mathrm{~kg}$, and $0.352 \pm 0.012 \mathrm{~kg}$, for GMD_D12, and $176.82 \pm 1.86 \mathrm{~kg}$, $190.59 \pm 2.07 \mathrm{~kg}, 223.30 \pm 2.31 \mathrm{~kg}$, and $214.12 \pm 2.31 \mathrm{~kg}$, for P12M.
\end{abstract}

Key Words: beef cattle, crossbreeding, post-weaning weight gain, yearling weight

\section{Introdução}

As características pós-desmama são muito importantes no estudo do desempenho ponderal de bovinos de corte manejados a campo, pois é nesta fase que os animais podem desenvolver seu potencial genético para crescimento, sem influência dos efeitos maternos. Excelência em velocidade de crescimento e eficiência alimentar é uma busca contínua para que os empreendimentos dedicados à produção de bovinos de corte nos trópicos alcancem eficiência econômica. As características inerentes aos trópicos, principalmente condições de estresse calórico e menor potencial de nutrientes das forrageiras em relação àquelas de clima temperado, são fatores que contribuem para que as empresas de pecuária de corte nestas regiões mantenham essencialmente rebanhos Zebus, principalmente o Nelore. MUJICA et al. (1997) comentam serem poucos os genótipos bovinos adaptados aos trópicos, com predominância das raças Zebu. De acordo com TURNER (1980), adaptação é um termo amplo usado para descrever a habilidade dos animais para se ajustarem às condições ambientes ou para inferência de modificações genéticas que tornam os animais mais preparados para existência em condições de meio específicas,

\footnotetext{
1 Projeto financiado pelo IAPAR/Programa Produção Animal.

2 Pesquisador do IAPAR, Polo Regional de Curitiba, Cx. Postal 2301, CEP 80011-970, Curitiba/PR. E.mail: dperotto@pr.gov.br

3 Prof. Adjunto, curso de Agronomia UFMS, CEP 79804-970, Cx. Postal 533, Dourados/MS. E.mail: acubas@ceud.ufms.br

4 Pesquisador do IAPAR, Est. Experimental de Paranavaí, CEP 87701-970, Cx. Postal 564, Paranavaí/PR. E.mail: cpviapar@celepar.gov.br

5 Engo-Agro., Rua Walter Hubacher 247-A, CEP 79750-000, Nova Andradina, MS.
} 
sendo o gado Zebu amplamente reconhecido como adaptável aos ambientes tropicais e subtropicais.

O gado Nelore, embora possua características altamente favoráveis à produção em condições de trópicos e subtrópicos, como a resistência ao estresse calórico e aos parasitos externos e internos, eficiência de produção em condições mínimas de manejo, bem como facilidade de parto a campo, caracteriza-se também em função de algumas características deficientes na eficiência reprodutiva e velocidade de crescimento. Parte desta situação do gado Nelore pode ser explicada pelo fato de ainda haver poucos programas de avaliação genética para a raça, o que a tem impedido de receber os benefícios oriundos da seleção. No entanto, programas pioneiros, embora ilhas isoladas de excelência, estão mudando este panorama, ao oferecer ao mercado reprodutores Nelore avaliados geneticamente com capacidade para contribuir com DEPs (diferenças esperadas nas progênies) positivas para características economicamente importantes, tais como precocidade sexual, fertilidade e velocidade de crescimento.

PASCHAL et al. (1995) compararam o desempenho pós-desmama do Aberdeen Angus com várias raças Zebu na Estação Experimental de Agricultura do Texas (Gray Brahman, Gir, Indu-Brasil, Nelore e Red Brahman), encontrando melhor desempenho pósdesmama para as raças Zebu, que não diferiram entre si em relação ao Angus. O peso a um ano foi maior para as duas raças Brahman, seguidas das três raças Zebu brasileiras (sem diferenças entre elas), sendo o menor peso o do Angus.

Este artigo resulta de dados coletados em um experimento de cruzamentos entre raças bovinas de corte, tendo como base matrizes da raça Nelore, com o objetivo de oferecer aos produtores resultados alternativos à produção de carne exclusiva com animais Nelore, visando à exploração de possíveis benefícios oriundos da heterose e complementariedade entre as raças.

\section{Material e Métodos}

Foram analisadas 634 observações de ganho médio diário de peso da desmama aos 12 meses (GMD_D12) e de pesos aos 12 meses de idade $(\mathrm{P} 12 \mathrm{M})$ de bezerros Nelore $(\mathrm{N}), 1 / 2$ Guzerá + $1 / 2$ Nelore $(\mathrm{GN}), 1 / 2$ Red Angus $+1 / 2$ Nelore $(\mathrm{RN})$ e $1 / 2$ Marchigiana $+1 / 2$ Nelore (MN), machos e fêmeas, nascidos no período de 1985 a 1997, oriundos de um experimento planejado de cruzamentos de animais da raça Nelore, realizado na Estação Experimental de Paranavaí, do INSTITUTO AGRONÔMICO DO PARANÁ - IAPAR, Região Noroeste do Paraná, cujo clima é enquadrado como Cfa, na classificação de Köeppen (IAPAR, 1974), com solos dos grupos Latosol Vermelho Amarelo (MINISTÉRIO DA AGRICULTURA, 1970). Para ambas as características, a amostra continha dados de 198 bezerros $\mathrm{N}$, $149 \mathrm{GN}, 151 \mathrm{RN}$ e $136 \mathrm{MN}$.

Os bezerros nascidos no período de 13 anos foram gerados via inseminação artificial, com sêmen de 91 touros (36 Nelore, 19 Guzerá, 15 Red Angus e 21 Marchigiana), adquirido de maneira aleatória, de acordo com a disponibilidade de sêmen de cada raça envolvida no programa nas centrais de inseminação artificial a cada ano, com a garantia de participação de pelo menos cinco touros de cada raça/ano, com pelo menos um touro sendo repetido, para garantia da conectabilidade dos dados gerados. Duas estações de inseminação foram utilizadas cada ano, de $1 \underline{0}$ de

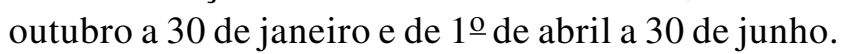
As vacas foram mantidas em pastagens de brachiaria (Brachiaria humidicula e Brachiaria decumbens), com suplementação no período seco via fornecimento de capim-napier (Pennisetum purpureum) e canade-açúcar (Saccharum officinarum) picados, pastejo direto em bancos de proteínas (Cajanus indicus e Leucaena leucocephala), além de uréia adicionada à mistura mineral. Os bezerros foram desmamados aproximadamente aos sete meses de idade. O rebanho foi submetido a rigoroso manejo sanitário, incluindo desverminações e pulverizações em épocas estratégicas para o controle de endo e ectoparasitoses, bem como vacinações sistemáticas contra febre aftosa, brucelose, carbúnculo hemático e paratifo dos bezerros. As variáveis peso aos 12 meses de idade e ganho médio diário de peso da desmama aos 12 meses de idade foram submetidas a ajuste prévio para a idade de 365 dias, por meio de coeficientes de regressão sobre a idade, estimados dentro de grupo genético, ano de nascimento e sexo do bezerro.

As observações de cada característica foram analisadas pelo método dos quadrados mínimos (SAS, 1985), de acordo com o seguinte modelo linear: $\mathrm{Y}_{\mathrm{ijk} l m n}=\mu+\mathrm{G}_{\mathrm{i}}+\mathrm{T}(\mathrm{G})_{\mathrm{ij}}+\mathrm{S}_{\mathrm{k}}+\mathrm{M}_{\mathrm{l}}+\mathrm{A}_{\mathrm{m}}+\mathrm{GS}_{\mathrm{ik}}+\mathrm{E}_{\mathrm{ijk} k \mathrm{mn}}$ em que $Y_{\text {ijklmn }}=$ valor observado para a característica (GMD_D12 e P12M) no n-ésimo animal, nascido no m-ésimo ano e l-ésimo mês, do k-ésimo sexo, progênie do j-ésimo touro, dentro do i-ésimo grupo genético; $\mu=$ média geral da característica $\mathrm{Y}$; $\mathrm{G}_{\mathrm{i}}=$ efeito fixo do i-ésimo grupo genético $(\mathrm{i}=1,2,3$, 
732 Rev. bras. zootec.

4); $\mathrm{T}(\mathrm{G})_{\mathrm{ij}}=$ efeito aleatório do j-ésimo touro $(\mathrm{j}=1,2$, $3, \ldots, n$ ), dentro do i-ésimo grupo genético (erro para testar $\mathrm{G}$ ); $\mathrm{S}_{\mathrm{k}}=$ efeito fixo do k-ésimo sexo do bezerro ( $\mathrm{k}=1$, macho e 2, fêmea); $\mathrm{M}_{1}$ = efeito fixo do l-ésimo mês de nascimento do bezerro $(1=1,2,3,4,7, \ldots, 12)$; $\mathrm{A}_{\mathrm{m}}=$ efeito fixo do m-ésimo ano de nascimento do bezerro $(m=1985, \ldots, 1998) ; \mathrm{GS}_{\mathrm{ik}}=$ efeito da interação entre oi-ésimo grupogenético o ok-ésimo sexo dobezerro; e $\mathrm{E}_{\mathrm{ijklmn}}=$ erro aleatório associado a cada observação.

\section{Resultados e Discussão}

O resumo das análises de variância das duas características avaliadas pode ser verificado na Tabela 1. Tanto para GMD_D12 $(\mathrm{P}<0,01)$ quanto para P12M $(\mathrm{P}<0,05)$, o efeito de mês de nascimento dos bezerros foi importante fonte de variação. Ao contrário do que foi verificado na fase pré-desmama (CUBAS et al., 2001), na qual os bezerros nascidos nos primeiros meses da estação de nascimentos de primavera/verão (julho a setembro) tiveram maiores ganhos e desmamaram mais pesados, na etapa entre a desmama e os 12 meses de idade os referidos animais tiveram os piores desempenhos em relação aos animais nascidos nos demais meses, embora com apenas algumas diferenças significativas $(\mathrm{P}<0,05)$ entre alguns meses de nascimento paraGMD_D12 e praticamente ausência de significância nas diferenças entre meses de nascimento para P12M.

Ano de nascimento do bezerro explicou $(\mathrm{P}<0,01)$ as diferenças de desenvolvimento verificadas nas duas características avaliadas, indicando grande variação dos fatores edafo-climáticos entre anos, já que as diferenças entre os diferentes anos do experimento aconteceram de maneira totalmente errática. Importantes efeitos fixos de mês e ano de nascimento do bezerro, causando variabilidade no desenvolvimento ponderal de bovinos de corte, têm sido relatados (ALENCAR et al., 1995, ALENCAR et al., 1997; ALENCAR et al., 1998; EUCLIDES FILHO et al., 1998; MAZZA, 1984; PEROTTO et al., 1998).

Assim como para as características pré-desmama, também foi importante o efeito de sexo no período pós-desmama $(\mathrm{P}<0,01)$, com os machos ganhando em média $51 \mathrm{~g}$ a mais por dia e pesando $17,06 \mathrm{~kg}$ a mais que as fêmeas. As médias de GMD_D12 e $\mathrm{P} 12 \mathrm{M}$, respectivamente, foram de $0,339 \pm 0,008 \mathrm{e}$ $209,74 \pm 1,51 \mathrm{~kg}$ para os machos e $0,288 \pm 0,007 \mathrm{e}$ $192,68 \pm 1,48 \mathrm{~kg}$ para as fêmeas. O efeito aleatório de touro dentro de cada grupo genético foi significativo para GMD_D12 $(\mathrm{P}<0,05)$ e $\mathrm{P} 12 \mathrm{M}(\mathrm{P}<0,01)$, indicativo de marcantes diferenças entre touros dentro de cada raça. Expressivas diferenças entre raças de touros $(\mathrm{P}<0,01)$ ocorreram e podem ser melhor visualizadas na Tabela 2, em que são descritas as médias e os

Tabela 1 - Resumo das análises de variância de características ponderais em bovinos Nelore e cruzas com Nelore, em Paranavaí-PR

Table 1 - Summary of analyses of variance of growth traits in crossbred and purebred Nellore cattle, in Paranavaí-PR

\begin{tabular}{|c|c|c|c|}
\hline \multirow[t]{2}{*}{$\begin{array}{l}\text { Fonte de variação } \\
\text { Source of variation }\end{array}$} & \multirow[t]{2}{*}{$\begin{array}{l}\mathrm{gl} \\
d f\end{array}$} & \multicolumn{2}{|c|}{$\begin{array}{l}\text { Valor do F computado } \\
\text { Computed } F \text { value }\end{array}$} \\
\hline & & GMD_D12 1 & $\mathrm{P} 12 \mathrm{M}^{1}$ \\
\hline $\begin{array}{l}\text { Raça ou grupo genético } \\
\text { Genetic group or breed }\end{array}$ & 3 & $39,92 * * *$ & $147,86 * * *$ \\
\hline $\begin{array}{l}\text { Touro (Grupo genético) } \\
\text { Sire (Genetic group) }\end{array}$ & 87 & $1,08 * *$ & $1,67 * * *$ \\
\hline $\begin{array}{l}\text { Sexo do bezerro } \\
\text { Calf sex }\end{array}$ & 1 & $50,52 * * *$ & $146,76^{* * *}$ \\
\hline $\begin{array}{l}\text { Grupo genético x sexo do bezerro } \\
\text { Genetic group x calf sex }\end{array}$ & 3 & $7,32 * * *$ & $4,90 * * *$ \\
\hline $\begin{array}{l}\text { Ano de nascimento do bezerro } \\
\text { Calf birth year }\end{array}$ & 12 & $37,28 * * *$ & $26,55 * * *$ \\
\hline $\begin{array}{l}\text { Mês de nascimento do bezerro } \\
\text { Calf birth month } \\
\mathrm{R} \text { ao quadrado }\end{array}$ & 9 & $7,41 * * *$ & $2,11 * *$ \\
\hline $\begin{array}{l}\text { R square } \\
\mathrm{CV}(\%)\end{array}$ & & $\begin{array}{r}0,71 \\
27,35\end{array}$ & $\begin{array}{l}0,77 \\
8,10\end{array}$ \\
\hline
\end{tabular}

${ }_{1} \mathrm{P} 12 \mathrm{M}=$ peso aos 12 meses de idade; GMD_D12 = ganho médio diário de peso da desmama aos 12 meses de idade; ${ }^{* *}=\mathrm{P}<0,05 ;{ }^{* * *}=\mathrm{P}<0,01$.

${ }^{1} P 12 M=$ yearling weight; GMD_D12 = average daily gain from weaning to yearling; ${ }^{* *}=P<.05 ;{ }^{* \star *}=P<.01$. 
Tabela 2 - Médias dos quadrados mínimos e respectivos erros-padrão de características ponderais em bovinos Nelore e cruzas com Nelore, em Paranavaí-PR

Table 2 - Least squares means and respective standard errors of growth traits in crossbred and purebred Nellore cattle, in Paranavaí-PR

\begin{tabular}{|c|c|c|}
\hline \multirow[t]{2}{*}{$\begin{array}{l}\text { Grupo genético } \\
\text { Genetic group }\end{array}$} & \multicolumn{2}{|c|}{$\begin{array}{c}\text { Médias dos quadrados mínimos } \\
\text { e erros-padrão } \\
\text { Least square means and } \\
\text { standard errors }\end{array}$} \\
\hline & GMD_D12 $(\mathrm{kg})^{1}$ & $\mathrm{P} 12 \mathrm{M}(\mathrm{kg})^{1}$ \\
\hline Nelore & $0,247 \pm 0,009$ & $176,82 \pm 1,86$ \\
\hline Guzerá x Nelore & $0,287 \pm 0,010$ & $190,59 \pm 2,07$ \\
\hline Red Angus x Nelore & $0,366 \pm 0,010$ & $223,30 \pm 2,03$ \\
\hline Marchigiana x Nelore & $0,352 \pm 0,012$ & $214,12 \pm 2,31$ \\
\hline
\end{tabular}

respectivos erros-padrão das características avaliadas para cada grupo genético.

Todas as diferenças entre grupos genéticos (Tabela 2) para GMD_D12 e P12M foram relevantes $(\mathrm{P}<0,01)$, com exceção daquela entre os grupos $\mathrm{RN}$ e MN para GMD_D12, que não foi significativa $(\mathrm{P}>0,05)$. Os maiores ganhos diários e pesos aos 12 meses de idade foram, respectivamente, dos grupos RN, MN, GN e Nelore. Diferenças entre grupos genéticos em trabalhos de cruzamento com base em rebanhos Nelore, detectando maiores pesos e ganhos de peso nos animais cruzados, têm sido reportadas (ALENCAR et al., 1995; PASCHAL et al., 1995; EUCLIDES FILHO et al., 1996; ALENCAR et al.,
1997; ALENCAR et al., 1998; EUCLIDES FILHO et al., 1998; MUNIZ e QUEIROZ, 1998).

As médias dos quadrados mínimos e respectivos erros-padrão de GMD_D12 e P12M, para cada sexo e grupo genético, são descritas na Tabela 3. Não houve diferenças importantes nos ganhos médios diários dos machos em relação às fêmeas nos grupos N (21 g) e GN (22 g), diferenças estas que foram significativas $(\mathrm{P}<0,01)$ nos grupos $\mathrm{RN}$ $(101 \mathrm{~g})$ e MN (60 g). Os machos pesaram significativamente mais que as fêmeas $(\mathrm{P}<0,01)$ aos 12 meses de idade, sendo as diferenças de $17,48 \mathrm{~kg}$ $(\mathrm{N}), 15,10 \mathrm{~kg}(\mathrm{GN}), 25,55 \mathrm{~kg}(\mathrm{RN})$ e $10,14 \mathrm{~kg}$ $(\mathrm{MN})$. A interação entre grupo genético e sexo do bezerro foi muito relevante $(\mathrm{P}<0,01)$ para ambas as características, em consequência do maior diferencial de dimorfismo sexual a favor dos machos no grupo RN em relação àquele dos demais grupos, conforme pode ser verificado nas Figuras 1 e 2 . Sob o aspecto fisiológico, possivelmente as condições de meio em que o trabalho foi conduzido, aliado ao fato de o gado Marchigiana ser mais tardio que o Red Angus, foram fatores capazes de explicar porquê os machos do grupo MN não puderam manifestar de maneira completa seu potencial de crescimento entre a desmama e os 12 meses de idade.

Os contrastes ortogonais comparando o desempenho entre Nelore e os demais grupos genéticos e entre RN com MN são descritos na Tabela 4. Os contrastes contêm informações importantes para a melhor compreensão das diferenças entre os grupos genéticos, além de se constituírem em

Tabela 3 - Médias ajustadas por quadrados mínimos e respectivos erros-padrão de características ponderais em bovinos Nelore e cruzas com Nelore, para machos e fêmas, em Paranavaí-PR

Table 3 - Least squares means and respective standard errors of growth traits in crossbred and purebred Nellore cattle, for males and females, in Paranavaí-PR

Raça do touro ou grupo genético

Sire breed or genetic group

Médias $\mathrm{QM}^{1}$ e erros-padrão $(\mathrm{kg})$

$L S^{1}$ means and standard errors

\begin{tabular}{lcr} 
& & P12M $^{2}$ \\
\cline { 2 - 3 } Nelore - machos (males) & GMD_D12 & $185,56 \pm 2,31$ \\
Nelore - fêmeas (females) & $0,258 \pm 0,012$ & $168,08 \pm 2,19$ \\
Guzerá x Nelore - machos (males) & $0,237 \pm 0,011$ & $198,14 \pm 2,56$ \\
Guzerá x Nelore - fêmeas (females) & $0,298 \pm 0,013$ & $183,04 \pm 2,46$ \\
Red Angus x Nelore - machos (males) & $0,276 \pm 0,013$ & $236,08 \pm 2,62$ \\
Red Angus x Nelore - fêmeas (females) & $0,417 \pm 0,013$ & $210,53 \pm 2,32$ \\
Marchigiana x Nelore - machos (males) & $0,316 \pm 0,012$ & $219,19 \pm 2,55$ \\
Marchigiana x Nelore - fêmeas (females) & $0,382 \pm 0,013$ & $209,05 \pm 2,96$
\end{tabular}

$1 \mathrm{QM}=$ quadrados médios; ${ }^{2} \mathrm{GMD}$ D12 = ganho médio diário da desmama aos 12 meses; P12M = peso aos 12 meses de idade.

1 LS = least squares; ${ }^{2} G M D$ D12 = average daily gain from weaning to yearling; $P 12 M=$ yearling weigth. 
734 Rev. bras. zootec.
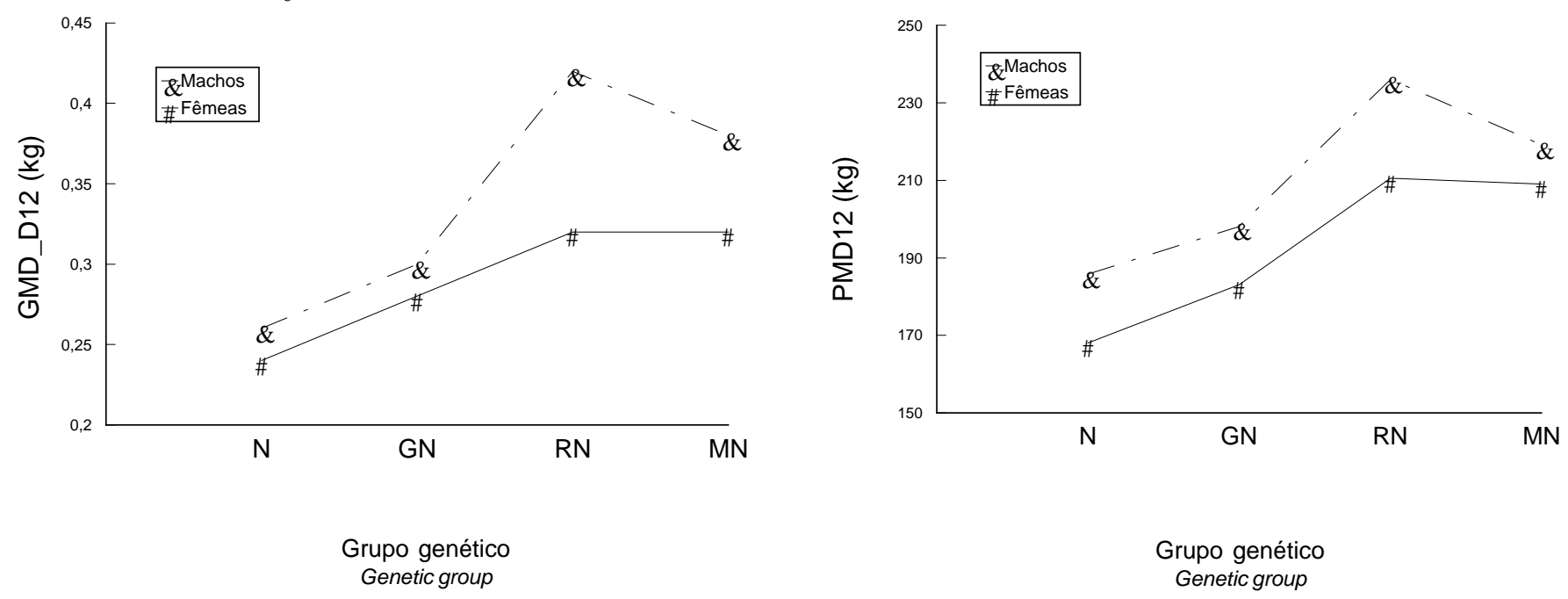

Figura 1 - Ganhos médios diários de peso dos bezerros (GMD D12), de acordo com o sexo e grupo genético, em que $\mathrm{N}=$ Nelore, $\mathrm{GN}=$ Guzerá $\mathrm{x}$ Nelore, $\mathrm{RN}=$ Red Angus $\mathrm{x}$ Nelore $\mathrm{e}$ $\mathrm{MN}=$ Marchigiana $\times$ Nelore.

Figure 1 - Calves average daily gain of weight, according to genetic group and sex, where $N=$ Nellore, GN = Guzerá $x$ Nellore, $R N=$ Red Angus $x$ Nellore $e$ $M N=$ Marchigiana $\times$ Nellore.

Figura 2 - Pesos aos 12 meses de idade dos bezerros (P12M), de acordo com o sexo e grupo genético, em que $\mathrm{N}=$ Nelore, $\mathrm{GN}=$ Guzerá $\times$ Nelore, $\mathrm{RN}=$ Red Angus $\times$ Nelore e MN = Marchigiana $\times$ Nelore.

Figure 2 - Calves yearling weights (P12M), according to genetic group and sex, where $N=$ Nellore, $G N=$ Guzerá $x$ Nellore, $R N=$ Red Angus $\times$ Nellore e $M N=$ Marchigiana $\times$ Nellore.

Tabela 4 - Estimativas de contrastes ortogonais e respectivos erros-padrão em bovinos Nelore e cruzas com Nelore, em Paranavaí-PR

Table 4 - Estimates of orthogonal contrasts and respective standard errors in crossbred and purebred Nellore cattle, in Paranavaí-PR

\begin{tabular}{|c|c|c|}
\hline \multirow{2}{*}{$\begin{array}{l}\text { Contraste } \\
\text { Contrast }\end{array}$} & \multicolumn{2}{|c|}{ Estimativa e respectivo erro-padrão } \\
\hline & ${ }^{1} \mathrm{GMD} \mathrm{D} 12(\mathrm{~kg})$ & ${ }^{1} \mathrm{P} 12 \mathrm{M}(\mathrm{kg})$ \\
\hline Nelore $(\mathrm{N})$ - Guzerá x Nelore & $-0,03970 \pm 0,012 * * *$ & $-13,77 \pm 2,43 * * *$ \\
\hline Nelore - Red Angus x Nelore & $-0,11894 \pm 0,012 * * *$ & $-46,48 \pm 2,41 * * *$ \\
\hline Nelore - Marchigiana x Nelore & $-0,10511 \pm 0,013 * * *$ & $-37,30 \pm 2,69 * * *$ \\
\hline Red Angus x N-Marchigiana x N & $0,01383 \pm 0,014^{\mathrm{ns}}$ & $9,18 \pm 2,79 * * *$ \\
\hline
\end{tabular}

$1 G M D D 12=$ average daily gain of weight from weaning to yearling; $P 12 M=$ yearling weight; ${ }^{n s}=P>.05$ and ${ }^{* * *}=P<.01$.

instrumentos que permitem inferências a respeito da predição do futuro desempenho das progênies das raças envolvidas. São esperados, respectivamente, maior ganho diário de peso entre a desmama e um ano e maior peso a um ano de idade $(\mathrm{P}<0,01)$, em relação ao Nelore, para as progênies GN $(39,70 \mathrm{~g}$ e $13,77 \mathrm{~kg}), \mathrm{RN}(118,94 \mathrm{~g}$ e $46,48 \mathrm{~kg})$ e MN (105,11 g e 37,30 kg). Progênies RN, embora não ganhem mais peso $(13,83 \mathrm{~g} ; \mathrm{P}>0,05)$ nesta fase, deverão ter maior peso aos 12 meses $(9,18 \mathrm{~kg}$; $\mathrm{P}<0,01)$ do que os bezerros $\mathrm{MN}$, vantagem adquirida no período pré-desmama.

\section{Conclusões}

Vale destacar que mês de nascimento do bezerro continuou sendo importante efeito fixo de ambiente, atuando sobre as características pós-desmama, já que o produtor pode decidir em que meses os bezerros devem nascer, para se beneficiar com as melhores condições naturais, concentrando a estação de nascimentos nos meses que permitem a obtenção de maiores pesos à desmama.

O efeito fixo de grupo genético indicou melhor desempenho pós-desmama para o grupo Red Angus 
x Nelore, seguido de Marchigiana x Nelore e Guzerá $x$ Nelore, indicativo de que cruzamentos de raças de corte com matrizes Nelore são opções disponíveis para os criadores para melhorar o desempenho de seus rebanhos.

A importante interação grupo genético x sexo do bezerro, com a diferença a favor dos machos em relação às fêmeas $\mathrm{MN}$ diminuindo drasticamente em contraste com aquela verificada com os machos em relação às fêmeas $\mathrm{RN}$, sugere que, nas idades mais jovens, os cruzamentos de Nelore com raças britânicas imprimem maior precocidade que aqueles envolvendo raças continentais.

\section{Referências Bibliográficas}

ALENCAR, M.M., BARBOSA, P.F., TUlLIO, R.R. et al. 1995. Peso à desmama de bezerros da raça Nelore e cruzados Canchim x Nelore e Marchigiana x Nelore. R. Soc. Bras. Zootec., 24(6):917-925.

ALENCAR, M.M., TREMATORE, R.L., BARBOSA, P.F. et al. 1997. Desempenho de bezerros filhos de touros das raças Nelore e Canchim e cruzados (F1) Charolês x Nelore e Piemontês x Nelore. Rev. Bras. Zootec., 26(3):461-466.

ALENCAR, M.M., TREMATORE, R.L., OLIVEIRA, J.A. et al. 1998. Características de crescimento até a desmama de bovinos da raça Nelore e cruzados Charolês x Nelore. R. Bras. Zootec., 27(1):40-46.

CUBAS, A.C., PEROTTO, D., ABRAhÃo, J.J.S. 2001. Desempenho até a desmama de bezerros Nelore e cruzas com Nelore. Rev. bras. zootec., 30(3):694-701.

EUCLIDES FILHO, K., FIGUEIREDO, G. R., SILVA, L.C.O. et al. Pesos ao nascer e à desmama e ganho pré-desmama de Nelore e seus mestiços com Fleckvieh, Chianina, Charolês e Angus. In: REUNIÃO ANUAL DA SOCIEDADE BRASILEIRA DE ZOOTECNIA, 33, 1996. Fortaleza. Anais... Fortaleza: SBZ, 1996. v.1, p.164-166.
EUCLIDES FILHO, K., FIGUEIREDO, G.R., SILVA, L.C.O. et al. 1998. Idade aos $165 \mathrm{~kg}$ de peso vivo para progênies de Nelore, Fleckvieh, Chianina, Charolês, F1's e retrocruzas. R. Bras. Zootec., 27(5):899-905.

IAPAR. Cartas climáticas do Estado do Paraná. 1974. Londrina: Instituto Agronômico do Paraná. 49p (Documento 18).

MAZZA, M.C. Estudo das influências sobre o crescimento até um ano de idade em um rebanho Guzerá. Belo Horizonte/MG: UFMG, 1984. 44p. Dissertação (Mestrado em Zootecnia) Unviersidade Federal de Minas Gerais, 1984.

MINISTÉRIO DA AGRICULTURA. 1970. Levantamentos de reconhecimento dos solos do noroeste do Estado do Paraná. Rio de Janeiro: Ministério da Agricultura. 102p (Boletim Técnico 14).

MUJICA, F., MENCHACA, M.A., HAMMOND, T.A. et al. 1997. Comportamiento post-desdete de novillos Senepol, Hereford y Senepol x Hereford, en Broksville, Florida. Arch. Latinoam. Prod. Anim., 5(2):155-166.

MUNIZ, C.A.S.D., QUEIROZ, S.A. 1998. Avaliação do peso à desmama e do ganho médio de peso de bezerros cruzados, no Estado do Mato Grosso do Sul.R. Bras. Zootec, 27(3):504-512.

PASCHAL, J.C., SANDERS, J.O., KERR, J.L. et al. 1995. Postweaning and feedlot growth and carcass characteristics of Angus, Gray Brahman, Gir, Indu-Brasil, Nellore, and Red Brahman-sired F1 calves. J. Anim. Sci., 73:373-380.

PEROTTO, D., CUBAS, A.C., MOLETTA, J.L. et al. 1998. Pesos ao nascimento e à desmama e ganho de peso do nascimento à desmama de bovinos Charolês, Caracu e cruzamentos recíprocos. R. Bras . Zootec., 27(4):730-737.

SAS INSTITUTE INC. 1985. SAS User's guide: statistics. 5.ed., Cary. NC: SAS Inst. Inc. 956p.

TURNER, J.W. 1980. Genetical and biological aspects of Zebu adaptability. J. Anim. Sci., 50:1201-1205.

Recebido em: 08/02/00

Aceito em: 24/01/01 